Ciencia y Educación, Vol. 5, No. 1, enero-abril, 2021

ISSN (impreso): 2613-8794・ISSN (en línea): 2613-8808

DOI: https://doi.org/10.22206/cyed.2021.v5i1.pp131-145

\title{
Relaciones sociales y académicas en estudiantes de Diseño a través de la plataforma "Second Life"
}

\author{
Social and academic relations in Design students \\ through the "Second Life" platform
}

Santiago Uribe $e^{\mathrm{a}}$ ORCID: 0000-0002-3457-0365

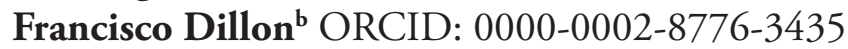

Recibido: 03/04/2020 - Aprobado: 03/07/2020

Cómo citar: Uribe, S., \& Dillon, F. (2021). Relaciones sociales y académicas en estudiantes de Diseño a través de la plataforma "Second Life". Ciencia y Educación, 5(1), 131-145. https://doi.org/10.22206/cyed.2021.v5i1.pp131-145

\section{Resumen}

El objetivo de este trabajo fue determinar si el entorno virtual de aprendizaje "Second Life" mejora las relaciones sociales y académicas de la materia de Diseño Básico Bidimensional de la carrera de Diseño Gráfico. El enfoque de la investigación fue de tipo cuantitativo pre experimental, de tipo secuencial exploratorio. La muestra del estudio correspondió a 12 estudiantes ( 8 hombres y 4 mujeres) de primer semestre de la carrera de Diseño Gráfico, en la Universidad Tecnológica Indoamérica, modalidad presencial de la ciudad de Quito, Ecuador. El trabajo concluye que, la utilización de la plataforma "Second Life" como entorno virtual y estrategia didáctica de aprendizaje mejora las relaciones sociales y el rendimiento académico en la materia de Diseño Básico Bidimensional de la población en estudio.

Palabras clave: análisis de redes sociales; docencia en entornos digitales; entorno virtual de aprendizaje; proceso de aprendizaje.

\begin{abstract}
The objective of this work was to determine if the virtual learning environment "Second Life" improves the social and academic relationships of the Basic Two-Dimensional Design subject of the Graphic Design degree. The research focus was quantitative pre-experimental, exploratory sequential type. The study sample corresponded to 12 students ( 8 men and 4 women) in the first semester of the Graphic Design degree program at the Universidad Tecnológica Indoamérica, face-to-face modality in the city of Quito, Ecuador. The work concludes that the use of the "Second Life" platform as a virtual environment and didactic learning strategy improves social relations and academic performance in the matter of Basic Two-Dimensional Design of the study population.
\end{abstract}

Keywords: Analysis of social networks; teaching in digital environments; virtual learning environment; learning process.

\footnotetext{
a Universidad Tecnológica Indoamérica, Ecuador. Correo-e: santiagouribe@uti.edu.ec

b Universidad Tecnológica Indoamérica, Ecuador. Correo-e: franciscodillon@uti.edu.ec
} 


\section{Introducción}

El uso de nuevas tecnologías de la información y comunicación (TIC y NTIC) ha servido como base para plantear cambios en los procesos de enseñanza y aprendizaje en el sistema educativo, en todos sus niveles. Esto se ve reflejado más que nada en las reformas curriculares y la implementación y uso de los Entornos Virtuales de Aprendizaje (EVA) como parte fundamental del aprendizaje escolar. La denominada sociedad de la información, al contar con nuevas herramientas tecnológicas y casi un libre acceso a la información, ha transformado la educación en un proceso dialéctico que se renueva, se transforma y adapta a los nuevos planteamientos didácticos, curriculares, $\mathrm{y}$ metodológicos de esta nueva era.

En el Foro Mundial de Educación realizado en China en el año 2015, se firmó la "Declaración de Qingdao” (Organización de las Naciones Unidas para la Educación, la Ciencia y la Cultura, 2015) que menciona en su declaratoria No. 13, la creación de nuevas vías de aprendizaje, siendo estas aplicadas desde la educación básica hasta la educación superior, a lo largo de toda una vida estudiantil. La importancia de las herramientas tecnológicas de información y comunicación recae en brindar una constante innovación por medio de la educación en línea, apoyándose en los entornos digitales, los entornos virtuales de aprendizaje, la gamificación, la ludificación y el aprendizaje significativo, los cuales, posibilitan un mayor índice de captación de la información.

Villarreal señala que: "la educación digital va más allá de la integración de tecnologías en los procesos formativos, pues requiere de la definición precisa de un propósito de aprendizaje concreto para el uso de la tecnología” (Villarreal, 2018, p. 2). Estas nuevas tecnologías, forman parte del desarrollo tecnológico; actualmente es más frecuente observar la implementación de las tecnologías de información y comunicación (TIC) en los entornos virtuales de aprendizaje (EVA), dentro y fuera de las aulas de clase.

Por otro lado, los autores Delgado y Solano (2009) mencionan que, tanto los docentes como los estudiantes aún no se encuentran familiarizados con el uso de este tipo de entornos, esto debido a que se requiere un nivel de destreza en el manejo de juegos y plataformas virtuales, al menos, en sus bases teóricas. Muchos de los docentes piensan que el utilizar entornos virtuales gamificados implica que sus estudiantes dejarán de lado los recursos de aprendizaje tradicionalmente usados, a través de los cuales, las planificaciones curriculares y las materias han sido diseñadas. Por ejemplo, la utilización de libros, las discusiones dentro del aula de clases respecto a un tema específico, la asistencia física a clases, entre otros; esto de alguna manera ha determinado que los docentes, y en menor grado los estudiantes, no puedan implementar estas estrategias como parte de su planificación curricular (Delgado y Solano, 2009).

El análisis de las relaciones sociales entendidas desde el punto de la interacción social, permiten a los miembros de una comunidad educativa identificar el tipo de relaciones académicas que establecen los miembros de una comunidad educativa y que determinan sus procesos de enseñanza y aprendizaje, en este caso, el modo virtual.

Los Entornos Virtuales de Aprendizaje (EVA, con un enfoque gamificado y ludificado), tienen como objetivo principal establecer estrategias creativas, que al ser aplicadas podrían generar mayor interés en los estudiantes que buscan experimentar con nuevos recursos y herramientas tecnológicas y que les permitan aprender de mejor manera. En este sentido, el canadiense "Murray Goldberg”, en el año de 1995 , miembro de la escuela de ingeniería en informática de la Universidad Británica de Columbia, creó una plataforma virtual a la que denominó "WebCT", la cual fue utilizada para investigar la efectividad del aprendizaje lector a través de la web. Desde su creación, "WebCT", o en español "WebCT- Corporación de Tecnologías Educativas", fue la primera plataforma en ser considerada como un recurso de ayuda a los procesos de enseñanza y aprendizaje, presente en alrededor de 80 países y utilizada por más o menos 4,000 universidades en todo el mundo. En el año 2006, aún en desarrollo, fue vendida a su competidor directo "Blackboard", y desde entonces, la misma ha desarrollado la idea original de 
"WebCT" como recurso y entorno virtual de aprendizaje (The University of British Columbia, 1995).

Martín (citado por Martí, 2006), en el año 2002, creó una plataforma de enseñanza virtual hecha con software libre, el cual fue llamado "Moodle" (Martí, 2016). Al momento, contaba con más de 81 millones de usuarios a nivel mundial. Esta es una plataforma educativa utilizada por estudiantes de todos los niveles educativos. "Moodle" significa "Modular Object Oriented Dynamic Learning Environment", en español: "Objeto Modular Orientado a un Entorno de Aprendizaje Dinámico”. Una de las utilidades y ventajas de esta plataforma es la facilidad al crear comunidades de aprendizaje en línea, las cuales, son gestionadas desde cada una de sus competencias curriculares y contextos.

A finales del año 2003, la empresa estadounidense originaria de San Francisco, California, "Linden Lab", creó una plataforma virtual en $3 \mathrm{D}$ de interacción en tiempo real, denominada: "Second Life" (Villavicencio, 2016). Esta plataforma utiliza avatares personalizados (representaciones gráficas de los usuarios de internet en un mundo creado digitalmente) de acuerdo a los gustos y preferencias de cada persona; sin límite alguno para crear y modificar la apariencia del avatar, desde un humano hasta un robot.

El diseño, versatilidad y dinamicidad de este entorno virtual ha sido utilizado con múltiples propósitos, por ejemplo, con fines de entretenimiento, de negocios, así como también para fines académicos y de desarrollo para el aprendizaje, al crear aulas y espacios completamente digitalizados, utilizados ahora por las universidades y centros de estudio para impartir clases y enviar trabajos que son realizados dentro del mismo entorno, como es el caso de la Universidad de Texas (en Norteamérica) y la Pontificia Universidad Católica del Perú (en Latinoamérica).

Según Torre y Romero (2018), la gamificación como una herramienta educativa, se presenta como un dilema para los profesores. Esto debido a la percepción docente relacionada al aporte de los juegos como parte del proceso de enseñanza y aprendizaje de sus estudiantes; que no ven como estrategias significativas de desarrollo educativo. El libro intenta sobre todo incor- porar acciones planteadas desde la teoría y la práctica que permitan a los docentes la incorporación de actividades gamificadas y ludificadas en el proceso de enseñanza y aprendizaje, bajo la utilización de las TIC y NTIC en y de las herramientas de la web 2.0 y 3.0 (principalmente el uso de los EVA).

Según Aguilera, Fúquene y Ríos (2014), los avances en tecnologías de la información, la proliferación del uso de redes sociales, además de la masificación de teléfonos inteligentes y tabletas; son considerados como technology enablers; los cuales, han propiciado las condiciones necesarias que posibilitan la implementación de esta técnica en entornos de aprendizaje. En este sentido, se presenta una breve revisión de la literatura relacionada, una descripción de la herramienta utilizada, la plataforma Duolingo ${ }^{\mathrm{TM}}$, y finalmente, se dejó abierta la discusión sobre los potenciales usos que tiene esta técnica en ambientes académicos.

Moreno, Leiva y Matas (2016) realizaron una revisión y análisis de aplicaciones y programas disponibles para diferentes dispositivos y sistemas operativos, planteando posibles propuestas de actividades con TIC, para su aplicación en el ámbito de la enseñanza de idiomas desde un enfoque comunicativo, a través de la gamificación y el Mobile Learning. Acorde con las diferentes ediciones del informe NMC Horizon Report, la implementación en el aula de las tecnologías basadas en la realidad aumentada, la gamificación y el Mobile Learning, supondrán una oportunidad para reconfigurar la práctica educativa acorde con las nuevas características, demandas y necesidades del estudiante diverso al que se atiende (Moreno et al., 2016).

Arroyo et al. (2018) mencionan que, el empleo de los entornos virtuales de aprendizaje supone un cambio de paradigma en las formas en las que se realiza el proceso docente universitario. En Ecuador, existen grandes potencialidades para el uso de entornos virtuales de aprendizaje en la universidad; sin embargo, la mayor parte de los docentes desconoce los beneficios de las plataformas y se rechaza su uso. Es por ello que en este estudio se plantearon como objetivos: 1) examinar aspectos teóricos sobre los entornos virtuales de aprendizaje en el contexto 
universitario y, 2) identificar las características de los entornos virtuales de aprendizaje en el Ecuador (Arroyo et al., 2018).

Para la obtención de resultados, además, se plantearon métodos en los niveles teórico y empírico, donde se determinaron las características y beneficios de dichos entornos y se analizaron las ventajas que traería su uso intensivo en la comunidad de práctica de docentes universitarios del Ecuador; llegando a la conclusión de que los EVA facilitan los procesos pedagógicos y académicos en los estudiantes y, además, posibilitan la creación de comunidades de práctica virtual.

La gamificación (del término gamification), consiste en el uso de técnicas, elementos y dinámicas propias de los juegos y el ocio en actividades de tipo no recreativas, con el fin de potenciar la motivación. Sirve además para reforzar la conducta del individuo, para solucionar un problema, mejorar la productividad, obtener un objetivo, activar el aprendizaje y evaluar a individuos concretos (Melo-Solarte y Díaz, 2018). La integración del juego en el sistema educativo ecuatoriano $y$, principalmente en el proceso de enseñanza y aprendizaje, une de manera efectiva el concepto de realidad aumentada; esta, es la visualización directa o indirecta de elementos del mundo real combinados o aumentados con elementos virtuales generados por un ordenador, cuya fusión da lugar a una realidad mixta, donde se considera a la misma como uno de los ejes fundamentales sobre el cuál, la gamificación, los procesos de enseñanza M-Learning (aprendizaje móvil) y E-Learning (educación virtual) cobran sentido, y sobre los cuales, se ha realizado esta investigación.

Algunas de las causas y efectos identificados que han permitido llegar a esta conclusión, se relacionan con los procesos de enseñanza convencional utilizados en la materia de Diseño Básico Bidimensional (véase figura 1).

Figura 1

Árbol de problemas

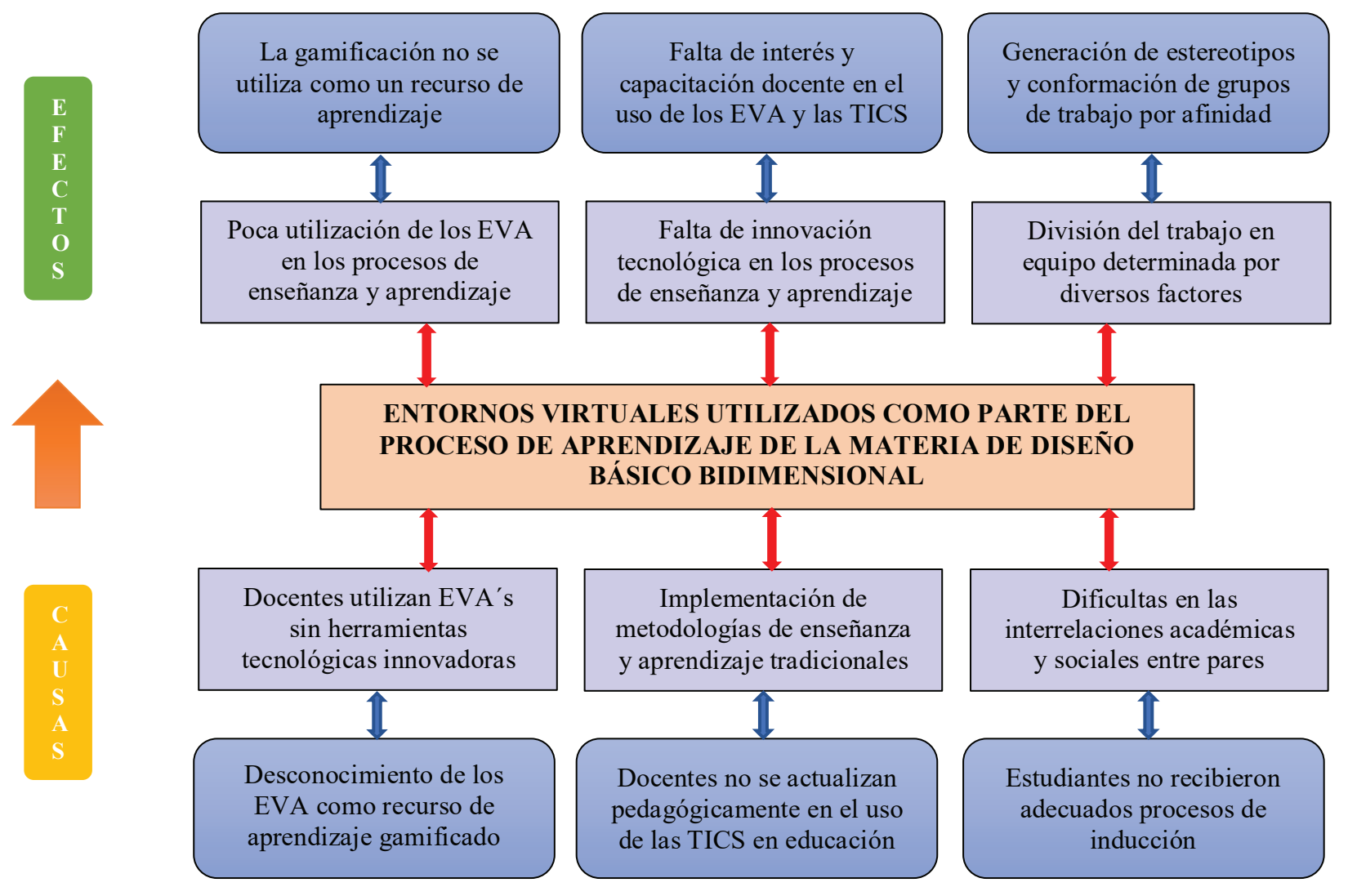




\section{Metodología}

\subsection{Objetivo e hipótesis}

El objetivo principal de la investigación fue identificar si el uso de los EVA con enfoque gamificado, en el caso de la plataforma "Second Life", ayuda a los procesos de aprendizaje y mejora las relaciones sociales y académicas de los estudiantes de la carrera de Diseño Gráfico del primer semestre de la modalidad presencial, de la Universidad Indoamérica, Sede Quito, Ecuador. Las hipótesis planteadas en esta investigación, fueron las siguientes:

\section{H1}

La utilización de la plataforma "Second Life" como entorno virtual y estrategia didáctica de aprendizaje mejora las relaciones sociales y el rendimiento académico en la materia de Diseño Básico Bidimensional de la población investigada.

Ho

La utilización de la plataforma "Second Life" como entorno virtual y estrategia didáctica de aprendizaje, no mejora las relaciones sociales y el rendimiento académico en la materia de Diseño Básico Bidimensional de la población investigada.

\subsection{Enfoque de la investigación}

El enfoque de investigación fue cuantitativo pre experimental, de tipo secuencial exploratorio (Johnson y Onwuegbuzie, 2004). Se realizaron procesos de análisis descriptivos, exploratorios y correlacionales para analizar las variables de estudio y verificar las hipótesis planteadas (Hérnandez-Sampieri et al., 2018; Padua, 2018).

\subsection{Población}

La población de estudio total de la carrera de Diseńo Gráfico corresponde a 125 estudiantes de los diferentes semestres. Debido al tipo y paradigma de investigación, se seleccionó una muestra de estudio de tipo no probabilística por conveniencia (Padua, 2018), compuesta por 12 estudiantes (8 hombres y 4 mujeres), en un rango de edad entre los 18 a 22 años, de primer semestre de la carrera de Diseño Gráfico, en el periodo académico B19 de la Universidad Tecnológica Indoamérica, modalidad presencial, de la ciudad de Quito, que compartían la asignatura de Diseño Básico Bidimensional.

\subsection{Instrumentos de investigación}

Los instrumentos de investigación fueron un cuestionario de doce preguntas de selección múltiple y una matriz de adyacencia, para poder determinar la información de las relaciones sociales y académicas de la población de estudio. Esta última es una matriz de doble entrada con números "0" y "1" que identifican la existencia o no de algún tipo de relación entre las personas con sus pares. En la tabla 1 se puede observar las relaciones hipotéticas planteadas en este ejemplo, para comprender un poco más lo expresado:

Se ha planteado las relaciones sociales entre Juan, Pedro y María. Juan se lleva muy bien con Pedro, pero no con María, por lo que en la celda JuanPedro, debería haber un número " 1 " y en la celda Juan-María, un número " 0 ". De esta misma manera, se ha llenado el resto de las relaciones hipotéticas de los miembros integrantes de este grupo; sin embargo, se debe mencionar que en las celdas en las que se encuentra el mismo nombre (JuanJuan), la misma debe ser llenada con números ceros, ya que lo que se pretende establecer, es el tipo de relación de una persona con otra, no el tipo de relaciones de una persona consigo mismo. 
Tabla 1

Ejemplo matriz de adyacencia

\begin{tabular}{|c|c|c|c|}
\hline Nombres & Juan & Pedro & María \\
\hline Juan & $\mathbf{0}$ & 1 & 0 \\
\hline Pedro & 1 & $\mathbf{0}$ & 1 \\
\hline María & 0 & 1 & $\mathbf{0}$ \\
\hline
\end{tabular}

Con la construcción de esta matriz de adyacencia, se buscaba caracterizar las relaciones sociales y académicas de la población, y cómo la topología de la red (dinámica de las relaciones sociales y académicas) se modifica o cambia en función de actividades específicas planteadas.

Por su parte, el cuestionario contó con una validación de expertos de dos profesionales especialistas en educación y uno en teoría de redes sociales. Posteriormente, se realizó un segundo proceso de validación de este mismo instrumento a través del Kappa de Cohen y Fleiss, que determinó en relación con los criterios de validación generales del instrumento que el resultado es excelente $($ Kappa $=1.0)$, en relación con los criterios específicos es aceptable (Kappa $=0.65)$; y en relación con la aplicación de la encuesta de manera general es excelente $($ Kappa $=1.0)$. La confiablidad de la escala utilizada en el cuestionario, una vez validado, fue muy confiable con un Alfa Cronbach $=0.997$.

\subsection{Análisis de datos}

El análisis de la topología de la red social y académica se realizó en el periodo académico B19 (segundo período académico del año 2019), en 3 momentos:

- El primero correspondió a las relaciones sociales entre sus miembros (véase figura 2) previa a la utilización del entorno "Second Life".

- Se levantó la información de las relaciones académicas, es decir, como los estudiantes se relacionan al momento de realizar tareas, trabajos y deberes en la materia investigada (véase figura 3), previa también a la utilización del entorno "Second Life".

- Se levantó la información de cómo los estudiantes se relacionan académica y socialmente a través del uso de la plataforma y entorno virtual de aprendizaje "Second Life" (véase figura 4); esto, con la finalidad de calcular algunas medidas estadísticas y verificar posibles cambios en su topología.

\section{Figura 2}

Topología de la red social investigada

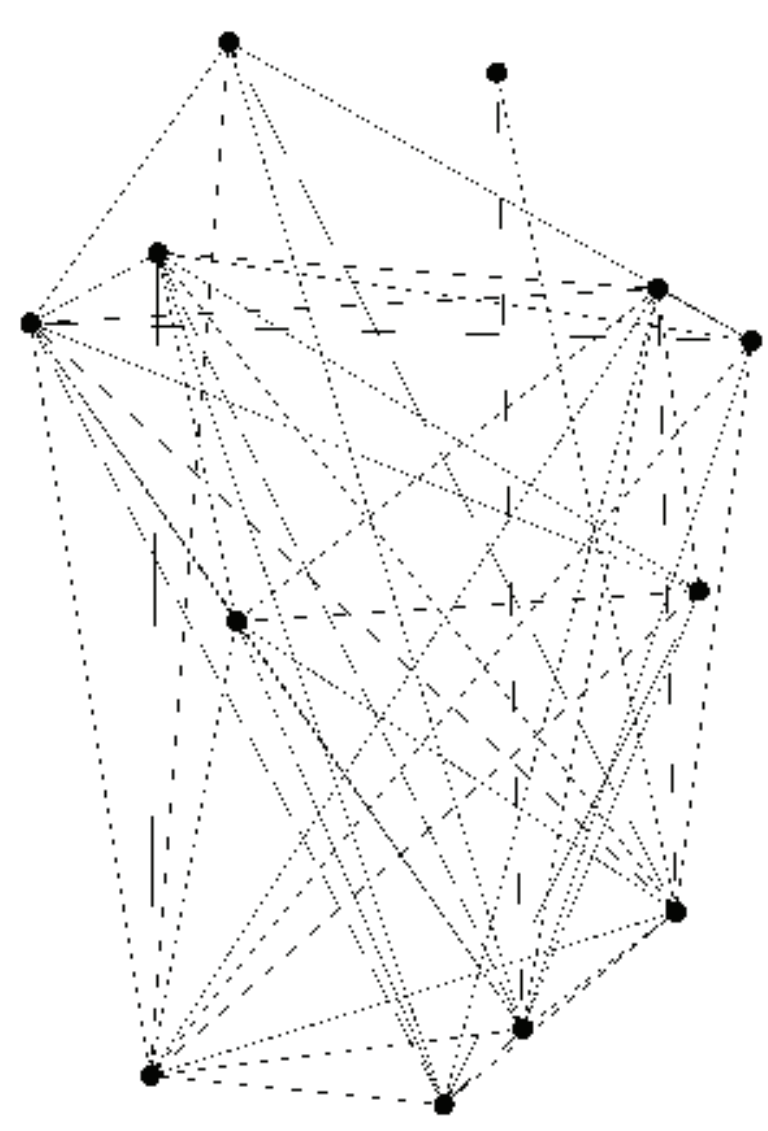

Figura 3

Topología de la red académica investigada

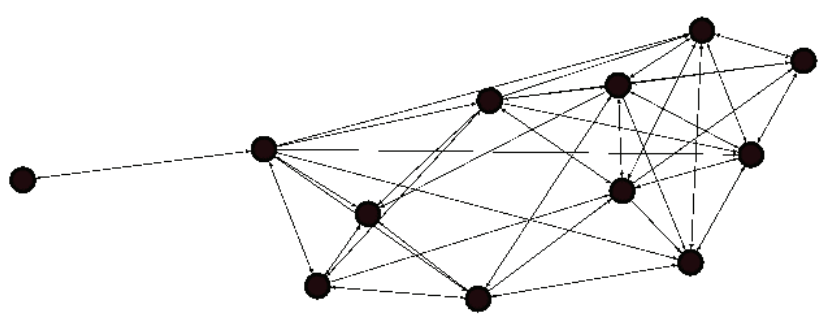


Figura 4

Topología de la red social y académica "Second Life" investigada

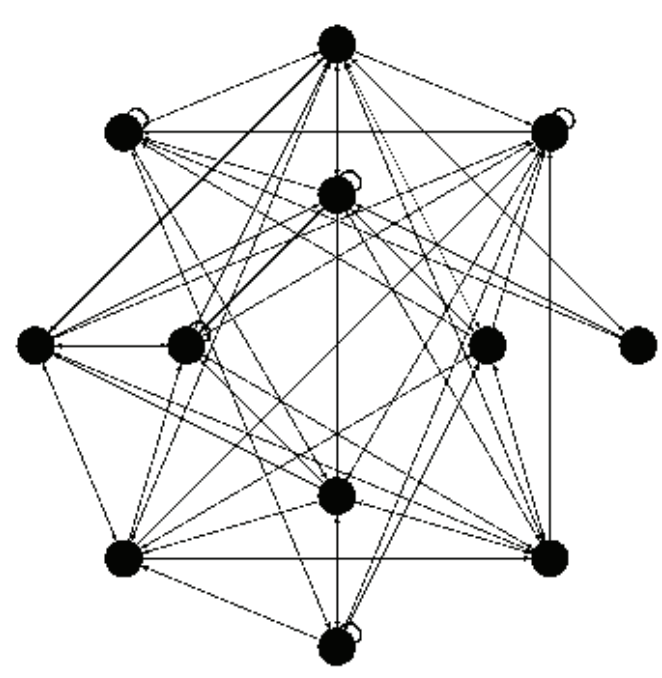

Una vez determinada la topología de la red, esta fue analizada a través del software de licencia libre, denominado "GEPHI", versión 0.9.2. Este es un software especializado en el análisis de redes sociales (ARS), dentro del cual, se pueden calcular de manera automática medidas estadísticas como: modularidad, pagerank, grados de entrada y salida, entre otras, necesarias para verificar si la topología de la red social, académica y del uso de la plataforma "Second Life" fueron modificadas. El análisis de los datos estadísticos fue realizado a través de los softwares estadísticos SPSS, versión 25 y Orange, versión. 3.25.

\section{Resultados}

\subsection{Análisis de la topología de la red de inte- racciones académicas y sociales}

Las medidas estadísticas dentro de la topología de la red que se han considerado estudiar para verificar si existió o no alguna modificación en su estructura, fueron: Modularidad, Pagerank, grados de entrada y salida, Betweenness y Eccentricity (Excentricidad).

Modularidad. También denominado algoritmo de comunidad; su cálculo ayuda a determinar la existencia de sub grupos dentro de una red y su topología (Park y Newman, 2004). En la tabla comparativa del cálculo de esta medida, en las tres redes, se pudo determinar que si existió una modificación de la misma (véase tabla 2), los números “ 0,1 y, 2", corresponden a la identificación y pertenencia del nodo a un determinado grupo en el caso de la red correspondiente al "Second Life", se puede observar que existe una mayor cohesión e identificación de ciertos nodos con otros nodos-grupos sociales o académicos, con los cuales en topologías anteriores, no existían relaciones.

Tabla 2

Modularidad

\begin{tabular}{|c|c|c|c|}
\hline & \multicolumn{3}{|c|}{ Modularidad } \\
\hline Código & Red social & $\begin{array}{c}\text { Red } \\
\text { académica }\end{array}$ & $\begin{array}{c}\text { Red luego de } \\
\text { utilizar second life }\end{array}$ \\
\hline A_1 & $\mathbf{0}$ & $\mathbf{0}$ & $\mathbf{0}$ \\
\hline A_2 & $\mathbf{2}$ & $\mathbf{1}$ & $\mathbf{1}$ \\
\hline A_3 & $\mathbf{0}$ & $\mathbf{1}$ & $\mathbf{0}$ \\
\hline A_4 & $\mathbf{0}$ & $\mathbf{0}$ & $\mathbf{1}$ \\
\hline A_5 & $\mathbf{0}$ & $\mathbf{0}$ & $\mathbf{0}$ \\
\hline A_6 & $\mathbf{1}$ & $\mathbf{1}$ & $\mathbf{0}$ \\
\hline A_7 & $\mathbf{1}$ & $\mathbf{0}$ & $\mathbf{1}$ \\
\hline A_8 & $\mathbf{1}$ & $\mathbf{0}$ & $\mathbf{1}$ \\
\hline A_9 & $\mathbf{1}$ & $\mathbf{1}$ & $\mathbf{1}$ \\
\hline A_10 & $\mathbf{2}$ & $\mathbf{1}$ & $\mathbf{0}$ \\
\hline A_11 & $\mathbf{2}$ & $\mathbf{1}$ & $\mathbf{0}$ \\
\hline A_12 & $\mathbf{2}$ & $\mathbf{1}$ & $\mathbf{1}$ \\
\hline
\end{tabular}

Pagerank. Es un algoritmo que permite calcular la popularidad de un nodo en la red (Park y Newman, 2004; Rubido, Grebogi y Baptista, 2017). Se puede observar que los más nodos populares, aunque distintos en las tres redes (abundantes principalmente en la red académica), fueron los nexos académicos con los demás nodos en la red que coadyuvaron a la elaboración del trabajo final de la materia de Diseño Básico Bidimensional utilizando como recurso el entorno de virtual de aprendizaje, la plataforma "Second Life". En la tabla comparativa del cálculo del page rank de las tres redes (véase tabla 3), la identificación del nodo más popular se encuentra marcado e identificado de manera visible en la tabla. 
Tabla 3

Pagerank

\begin{tabular}{|c|c|c|c|}
\hline & \multicolumn{3}{|c|}{ Pagerank } \\
\hline Código & Red social & $\begin{array}{c}\text { Red } \\
\text { académica }\end{array}$ & $\begin{array}{c}\text { Red luego de } \\
\text { utilizar second } \\
\text { life }\end{array}$ \\
\hline A_1 & 0.064795 & 0.055332 & 0.070639 \\
\hline A_2 & 0.067885 & $\mathbf{0 . 1 1 1 8 1 3}$ & 0.024183 \\
\hline A_3 & 0.062483 & 0.048335 & 0.090246 \\
\hline A_4 & 0.057223 & 0.052578 & 0.040183 \\
\hline A_5 & $\mathbf{0 . 0 9 0 0 6 5}$ & 0.08274 & 0.065023 \\
\hline A_6 & 0.07996 & 0.09139 & 0.08211 \\
\hline A_7 & 0.024588 & 0.016947 & 0.073281 \\
\hline A_8 & 0.074468 & 0.041678 & $\mathbf{0 . 1 1 9 4 0 3}$ \\
\hline A_9 & 0.071093 & $\mathbf{0 . 1 0 3 4 7 2}$ & 0.062002 \\
\hline A_10 & 0.065115 & $\mathbf{0 . 1 1 5 6 3 4}$ & 0.061215 \\
\hline A_11 & 0.069559 & $\mathbf{0 . 1 1 5 6 3 4}$ & 0.070428 \\
\hline A_12 & 0.055025 & 0.082807 & 0.07778 \\
\hline
\end{tabular}

Grados de entrada y salida. Algoritmos que permiten identificar la suma de las relaciones referidas hacia un actor por otros (grados de entrada) y, la suma de las relaciones que los actores dicen tener con el resto (grado de salida) (Rubido, Grebogi y Baptista, 2017). En relación a la tabla comparativa de los grados de entrada (véase tabla 4), no se ha identificado cambios significativos en su topología, mientras que en relación a la tabla comparativa de los grados de salida (ver Tabla 5), se puede observar que los nodos en la red han variado significativamente, esto como resultado de la nueva interconexión (establecimiento de nuevas relaciones) debido a la elaboración del trabajo final de la materia de Diseño Básico Bidimensional, utilizando como recurso el entorno de virtual de aprendizaje, la plataforma "Second Life".
Tabla 4

Grado de entrada

\begin{tabular}{|c|c|c|c|}
\hline & \multicolumn{3}{|c|}{ Grado de entrada } \\
\hline Código & Red social & Red académica & $\begin{array}{c}\text { Red luego de } \\
\text { utilizar second } \\
\text { life }\end{array}$ \\
\hline A_1 & 7 & $\mathbf{5}$ & $\mathbf{7}$ \\
\hline A_2 & $\mathbf{8}$ & 7 & $\mathbf{1}$ \\
\hline A_3 & 7 & $\mathbf{4}$ & 7 \\
\hline A_4 & $\mathbf{5}$ & $\mathbf{4}$ & $\mathbf{3}$ \\
\hline A_5 & $\mathbf{1 0}$ & $\mathbf{5}$ & $\mathbf{5}$ \\
\hline A_6 & $\mathbf{9}$ & $\mathbf{6}$ & $\mathbf{6}$ \\
\hline A_7 & $\mathbf{1}$ & $\mathbf{1}$ & 7 \\
\hline A_8 & $\mathbf{8}$ & $\mathbf{3}$ & $\mathbf{1 0}$ \\
\hline A_9 & $\mathbf{8}$ & $\mathbf{6}$ & $\mathbf{5}$ \\
\hline A_10 & 7 & 7 & $\mathbf{4}$ \\
\hline A_11 & $\mathbf{8}$ & $\mathbf{7}$ & $\mathbf{6}$ \\
\hline A_12 & $\mathbf{6}$ & $\mathbf{5}$ & $\mathbf{7}$ \\
\hline
\end{tabular}

Tabla 5

Grado de salida

\begin{tabular}{|c|c|c|c|}
\hline & \multicolumn{2}{|c|}{ Grado de salida } \\
\hline Código & $\begin{array}{c}\text { Red } \\
\text { social }\end{array}$ & $\begin{array}{c}\text { Red } \\
\text { académica }\end{array}$ & $\begin{array}{c}\text { Red luego de } \\
\text { utilizar second } \\
\text { life }\end{array}$ \\
\hline A_1 & $\mathbf{4}$ & $\mathbf{3}$ & $\mathbf{4}$ \\
\hline A_2 & 7 & $\mathbf{3}$ & $\mathbf{3}$ \\
\hline A_3 & $\mathbf{1 3}$ & $\mathbf{7}$ & $\mathbf{7}$ \\
\hline A_4 & $\mathbf{5}$ & $\mathbf{6}$ & $\mathbf{7}$ \\
\hline A_5 & $\mathbf{8}$ & $\mathbf{5}$ & $\mathbf{6}$ \\
\hline A_6 & $\mathbf{1 3}$ & $\mathbf{6}$ & $\mathbf{5}$ \\
\hline A_7 & $\mathbf{5}$ & $\mathbf{3}$ & $\mathbf{4}$ \\
\hline A_8 & $\mathbf{1 3}$ & $\mathbf{1 1}$ & $\mathbf{9}$ \\
\hline A_9 & $\mathbf{1 2}$ & $\mathbf{8}$ & $\mathbf{6}$ \\
\hline A_10 & $\mathbf{9}$ & $\mathbf{5}$ & $\mathbf{5}$ \\
\hline A_11 & $\mathbf{9}$ & $\mathbf{5}$ & $\mathbf{6}$ \\
\hline A_12 & $\mathbf{7}$ & $\mathbf{4}$ & $\mathbf{6}$ \\
\hline
\end{tabular}


Betweenness. Algoritmo que permite identificar los nodos en la red intermediarias en los procesos de comunicación con otros nodos dentro de la misma. Estos nodos, por sus características, pueden ser considerados nodos "líderes", ya que controlan el flujo de información que se transmite en la red (Albert y Barabási, 2002; Park y Newman, 2004; Rubido, Grebogi y Baptista, 2017). En relación con la tabla comparativa de esta medida estadística, se puede observar que el nodo (A_8) sirvió como uno de los intermediarios de las directrices emitidas por el investigador, relacionado con la utilización del entorno virtual de aprendizaje "Second Life" y los estudiantes. Se evidencia, además, que existe una mayor interacción entre los nodos de la red y su interconexión; la comunicación es mucho más fluida (véase tabla 6), y se identifican muchos más nodos intermediarios de comunicación que en las dos primeras redes.

Tabla 6

Betweenness

\begin{tabular}{|c|c|c|c|}
\hline & \multicolumn{3}{|c|}{ Betweenness } \\
\hline Código & Red social & Red académica & $\begin{array}{c}\text { Red luego de } \\
\text { utilizar second } \\
\text { life }\end{array}$ \\
\hline A_1 & 0.005417 & $\mathbf{0 . 1 6 0 5 5 6}$ & 0.52381 \\
\hline A_2 & 0.00497 & 0.018333 & 0.578947 \\
\hline A_3 & 0.028998 & 0.055646 & $\mathbf{0 . 7 3 3 3 3 3}$ \\
\hline A_4 & 0.005179 & 0.092279 & $\mathbf{0 . 6 8 7 5}$ \\
\hline A_5 & 0.035595 & 0.075703 & $\mathbf{0 . 6 8 7 5}$ \\
\hline A_6 & 0.075833 & 0.028481 & 0.647059 \\
\hline A_7 & 0.000595 & 0.052381 & 0.578947 \\
\hline A_8 & $\mathbf{0 . 1 6 0 6 9 4}$ & $\mathbf{0 . 2 7 4 8 1 9}$ & $\mathbf{0 . 8 4 6 1 5 4}$ \\
\hline A_9 & 0.046498 & $\mathbf{0 . 1 4 8 5 1 5}$ & $\mathbf{0 . 6 8 7 5}$ \\
\hline A_10 & 0.013472 & 0.017596 & 0.647059 \\
\hline A_11 & 0.014415 & 0.017596 & 0.647059 \\
\hline A_12 & 0 & 0.000952 & 0.647059 \\
\hline
\end{tabular}

Eccentricity (Excentricidad). Es una medida estadística que permite identificar el número de enlaces necesarios para conectar un nodo de la red, con otro más lejano dentro de la misma, (Albert y Barabási, 2002; Cestero y Mateos, 2018; Park y Newman, 2004; Rubido, Grebogi y Baptista, 2017; Velázquez y Aguilar, 2005). El promedio de pasos que los nodos deben dar en la red "Second Life", es de 2 pasos, salvo uno de los nodos que debería dar 3 para conectarse con cualquier nodo en la red. Este fenómeno se debe a que los flujos de comunicación, así como la interacción académica y social de la red, han permitido que los estudiantes puedan comunicarse más y mejor al utilizar el entorno virtual de aprendizaje "Second Life", como parte de la elaboración del trabajo final de la materia de Diseño Básico Bidimensional (véase tabla 7).

Tabla 7

Eccentricity (Excentricidad)

\begin{tabular}{|c|c|c|c|}
\hline & \multicolumn{3}{|c|}{ Eccentricity (excentricidad) } \\
\hline Código & $\begin{array}{c}\text { Red } \\
\text { social }\end{array}$ & Red académica & $\begin{array}{c}\text { Red luego de } \\
\text { utilizar second } \\
\text { life }\end{array}$ \\
\hline A_1 & 3 & 3 & 3 \\
\hline A_2 & 3 & 5 & 2 \\
\hline A_3 & 2 & 4 & 2 \\
\hline A_4 & 2 & 3 & 2 \\
\hline A_5 & 3 & 4 & 2 \\
\hline A_6 & 2 & 5 & 2 \\
\hline A_7 & 2 & 3 & 2 \\
\hline A_8 & 2 & $\mathbf{2}$ & $\mathbf{2}$ \\
\hline A_9 & $\mathbf{2}$ & $\mathbf{4}$ & $\mathbf{2}$ \\
\hline A_10 & $\mathbf{2}$ & 5 & $\mathbf{2}$ \\
\hline A_11 & $\mathbf{2}$ & $\mathbf{5}$ & $\mathbf{2}$ \\
\hline A_12 & 3 & 5 & \\
\hline
\end{tabular}




\subsection{Análisis correlacional}

El análisis correlacional de las medidas estadísticas calculadas en la red "Second Life" permitió comprobar la hipótesis afirmativa planteada en la investigación, ya que a través del estudio preliminar de la topología de la red realizado en esta investigación, se ha identificado que la utilización del entorno virtual de aprendizaje "Second Life”, sí modificó las relaciones tanto académicas como sociales dentro de la población de estudio.

En la figura 5, se puede observar que existe una relación directa y una correlación positiva muy alta $(\mathrm{r}=0.96)$, relacionado a la popularidad que tiene un nodo en la red y su grado de entrada, nuevamente se mencionará que este tiene relación directa con la utilización del entorno virtual de aprendizaje "Second Life" dentro de la elaboración del trabajo final de la materia de Diseño Básico Bidimensional (Watts, 2006).

\section{Figura 5}

Topología de la red social y académica "Second Life" investigada (Grado de entrada - pagerank)

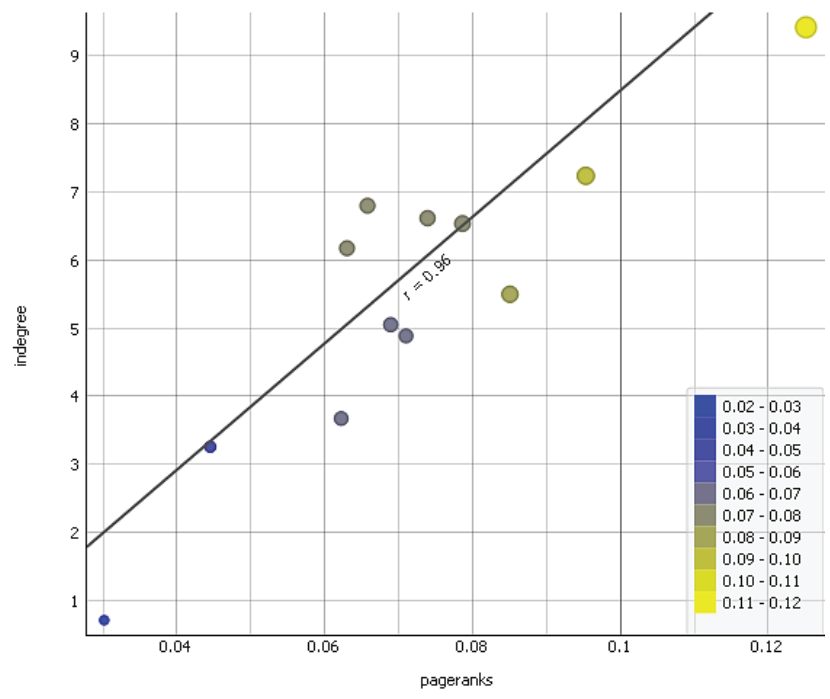

En la figura 6, se puede observar que existe una relación directa y una correlación positiva alta $(\mathrm{r}=0.76)$, relacionado a la popularidad que tiene un nodo en la red y la intermediación del mismo, dentro del flujo de comunicaciones que fluye dentro de la misma, en otras palabras, mientras más popular es un nodo en la red, mayor su probabilidad de manejar los flujos de infor- mación académica y social que se establece dentro de la misma.

\section{Figura 6}

Topología de la red social y académica "Second Life" investigada (Betweenness - pagerank)

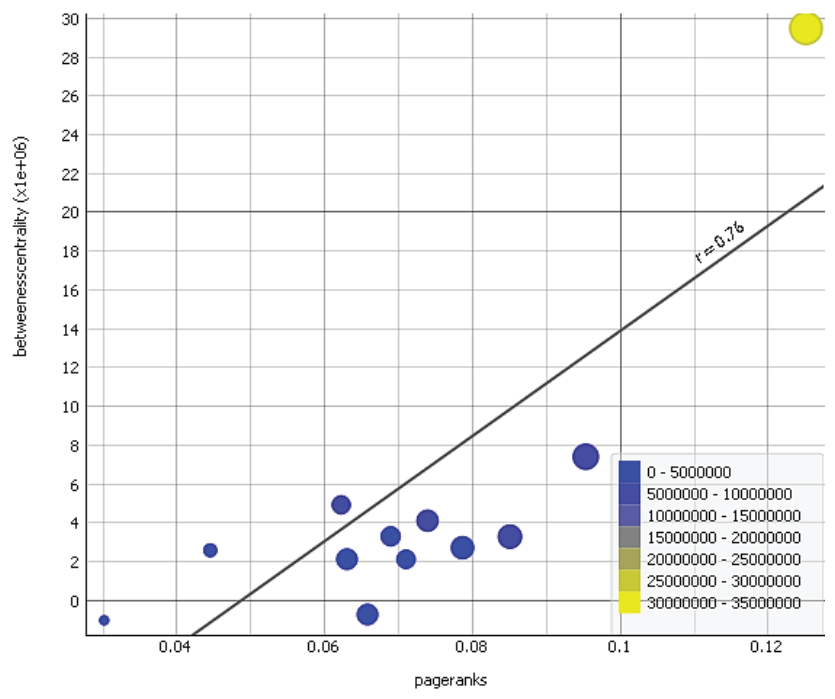

Adicionalmente, se ha podido determinar que la conformación de grupos académicos no se ve directamente influenciados por los grados de entrada, salida, la intermediación de los nodos dentro de la red, ni mucho menos, se ven afectados por la popularidad; lo que, además quiere decir que al existir adecuados espacios que propician la comunicación y relaciones sociales y académicas de los nodos en la red, mayor es la probabilidad de que los grupos conformados no presenten estereotipos o conductas que dificulten su comunicación.

Para comprobar la hipótesis afirmativa, se procedió a ingresar los datos obtenidos en la aplicación del cuestionario en el software SPSS versión 25, cuyos datos fueron utilizados como referencia en la comprobación de la hipótesis afirmativa relacionada al uso de la plataforma "Second Life" como entorno virtual y estrategia didáctica de aprendizaje, para mejorar las relaciones sociales y el rendimiento académico (véase tabla 8). El recuento de las respuestas esperadas, también fue analizada, esta permitirá confirmar o descartar la hipótesis nula o alternativa una vez calculado el chi cuadrado (véase tabla 9). 
Tabla 8

Chi cuadrado

\begin{tabular}{|c|c|c|c|c|c|c|}
\hline \multicolumn{7}{|c|}{ Resumen de procesamiento de casos } \\
\hline & \multicolumn{6}{|c|}{ Casos } \\
\hline & \multicolumn{2}{|c|}{ Válido } & \multicolumn{2}{|c|}{ Perdido } & \multicolumn{2}{|c|}{ Total } \\
\hline & $\mathbf{N}$ & Porcentaje & $\mathbf{N}$ & Porcentaje & $\mathbf{N}$ & Porcentaje \\
\hline $\begin{array}{l}\text { ¿El entorno virtual de aprendizaje } \\
\text { «Second Life”, permitió mejorar las } \\
\text { relaciones sociales con sus compañeros } \\
\text { en la materia de Diseńo Básico } \\
\text { Bidimensional? }\end{array}$ & \multirow{2}{*}{12} & \multirow{2}{*}{$100,00 \%$} & \multirow{2}{*}{0} & \multirow{2}{*}{$0,00 \%$} & \multirow{2}{*}{12} & \multirow{2}{*}{$100,00 \%$} \\
\hline $\begin{array}{c}\text { * ¿El entorno virtual de aprendizaje } \\
\text { "Second Life", permitió mejorar } \\
\text { sus interacciones académicas } \\
\text { (rendimiento) en la materia de Diseño } \\
\text { Básico Bidimensional? }\end{array}$ & & & & & & \\
\hline
\end{tabular}

Tabla 9

Recuento de casos

Tabla cruzada ¿El entorno virtual de aprendizaje "Second Life”, permitió mejorar las relaciones sociales con sus compañeros en la materia de Diseño Básico Bidimensional?* ¿El entorno virtual de aprendizaje "Second Life”, permitió mejorar sus interacciones académicas (rendimiento) en la materia de Diseńo Básico Bidimensional?

\begin{tabular}{|c|c|c|c|c|c|}
\hline & \multicolumn{2}{|c|}{$\begin{array}{l}\text { ¿El entorno virtual de aprendizaje «Second } \\
\text { Life”, permitió mejorar sus interacciones } \\
\text { académicas (rendimiento) en la materia de } \\
\text { Diseńo Básico Bidimensional? }\end{array}$} & \multirow[t]{2}{*}{ Total } \\
\hline & & & $\mathrm{NO}$ & SI & \\
\hline \multirow{4}{*}{$\begin{array}{l}\text { ¿El entorno virtual de aprendizaje «Second } \\
\text { Life”, permitió mejorar las relaciones } \\
\text { sociales con sus compañeros en la materia } \\
\text { de Diseño Básico Bidimensional? }\end{array}$} & \multirow[b]{2}{*}{$\mathrm{NO}$} & Recuento & 2 & 0 & 2 \\
\hline & & $\begin{array}{l}\text { Recuento } \\
\text { esperado }\end{array}$ & 0,3 & 1,7 & 2 \\
\hline & \multirow{2}{*}{ SI } & Recuento & 0 & 10 & 10 \\
\hline & & $\begin{array}{l}\text { Recuento } \\
\text { esperado }\end{array}$ & 1,7 & 8,3 & 10 \\
\hline \multirow{2}{*}{\multicolumn{2}{|c|}{$\begin{array}{c}\text { Total } \\
\text { Recuento esperado }\end{array}$}} & Recuento & 2 & 10 & 12 \\
\hline & & 2 & 10 & 12 & \\
\hline
\end{tabular}

El cálculo estadístico del chi cuadrado (véase tabla 9) arrojó que el nivel de significancia calculado es menor a $0.005(0.001<0.005)$; debido a esto, se descarta la hipótesis nula y se acepta la hipótesis alternativa: "La utilización de la plataforma "Second Life" como entorno virtual y estrategia didáctica de aprendizaje, mejora las relaciones sociales y el rendimiento académico en la materia de Diseño Básico Bidimensional de la población investigada". 
Tabla 9

Cálculo de chi cuadrado

\begin{tabular}{|c|c|c|c|c|c|}
\hline \multicolumn{7}{|c|}{ Pruebas de chi-cuadrado } \\
\hline & Valor & df & $\begin{array}{c}\text { Significación asintótica } \\
\text { (bilateral) }\end{array}$ & $\begin{array}{c}\text { Significación exacta } \\
\text { (bilateral) }\end{array}$ & $\begin{array}{c}\text { Significación exacta } \\
\text { (unilateral) }\end{array}$ \\
\hline Chi-cuadrado de Pearson & $12,000^{\mathrm{a}}$ & 1 & $\mathbf{0 , 0 0 1}$ & \\
\hline Corrección de continuidad & 5,88 & 1 & 0,015 & & \\
\hline Razón de verosimilitud & 10,813 & 1 & 0,001 & 0,015 & \\
\hline Prueba exacta de Fisher & & & & & 0,015 \\
\hline N de casos válidos & 12 & & & & \\
\hline \multicolumn{7}{|c|}{ b. 3 casillas (75,0\%) han esperado un recuento menor que 5. El recuento mínimo esperado es ,33. } \\
\hline
\end{tabular}

\section{Discusión y Conclusiones}

Los EVA, hoy más que nunca se encuentran en boga. Esto debido a la facilidad en el acceso a plataformas digitales, que facilitan su utilización y la ayuda que representa en el trabajo de planificación curricular docente, dentro y fuera del aula (Edel-Navarro, 2010). Son muchas las instituciones educativas que implementan estrategias relacionadas a mejorar el contenido pedagógico y las estrategias didácticas necesarias para complementar el proceso de enseñanza y aprendizaje, lo que es tomada como referencia en este estudio es la implementación de EVA gamificados, a través de los cuales, tanto docentes como estudiantes exploran nuevas alternativas al momento de enseñar y aprender, a través del uso de la tecnología y el juego (Villarreal, 2018; Villavicencio, 2016).

Los EVA (principalmente los gamificados), proporcionan al docente las herramientas necesarias para la implementación de las TIC y NTIC dentro del proceso de enseńanza y aprendizaje (Edel-Navarro, 2010). La utilización del EVA tomado como referencia en este estudio y denominado "Second Life", permitió determinar estadísticamente la influencia directa de la utilización de este entorno en el proceso de enseñanza y como estrategia complementaria en el proceso de aprendizaje de la materia de "Diseño Básico Bidimensional". De la misma manera, se identificó que su utilización mejora las relaciones sociales y académicas al modificar la topología de la red. Esto fue logrado principalmente debido a un proceso de investigación y análisis estadístico riguroso, tanto de los instrumentos de investigación como de la construcción del marco teórico y metodológico de referencia utilizado en esta investigación (Edel-Navarro, 2010; Norman, 2001; UNESCO, 2018; Villarreal, 2018).

Este trabajo permitió a los estudiantes de la carrera de Diseño Gráfico en el primer semestre, mejorar sus relaciones sociales en la materia de Diseño Básico Bidimensional, al establecer nuevos canales de comunicación en la elaboración de trabajos finales utilizando la plataforma "Second Life". Además, aportó de manera significativa a sus relaciones académicas, ya que, para mejorar la elaboración de estos trabajos, los estudiantes formaron nuevos grupos, para además, ayudó a mejorar su interacción en la plataforma tomada como referencia en esta investigación.

Existe una diferencia relevante al momento de analizar conceptualmente a la gamificación dentro del proceso educativo y el aprendizaje basado en juegos. Mientras el primero toma como base la dinámica de los juegos como un componente de motivación extrínseca en el aprendizaje, el segundo utiliza el juego y su dinámica como un recurso fundamental dentro del proceso de enseñanza y aprendizaje. Por ejemplo, no sería lo mismo tomar como referencia el juego denominado "Minecraft" para explicar la importancia de la programación y crear entornos virtuales en los estudiantes de Diseño Gráfico, que utilizar "Minecraft" para que los estudiantes del mismo contexto creen un 
espacio personalizado; o que dentro de la materia de Diseño Básico Bi y tridimensional se evalúe la utilización de los recursos del juego para crear dicho entorno. (Aguilera et al., 2014; Melo-Solarte y Díaz, 2018; Saraguro-Bravo et al., 2016)

La gamificación expande las posibilidades de generar prácticas innovadoras como el flipped classroom (o clase invertida), blended learning (aprendizaje semipresencial) y aprendizaje adaptativo; lo que permite al docente dedicar horas del plan formativo a la práctica y dejar el contenido teórico para el estudio individual del estudiante (Torre y Romero, 2018).

Las principales características de la gamificación aplicada en el aula, en el caso de la plataforma "Second Life", tomada como base metodológica y teórica de análisis en este trabajo de investigación; integra los conceptos de gamificación y del aprendizaje basado en juegos (adaptativo y significativo), ya que combina elementos de las TIC y las NTIC con la realidad aumentada y las prácticas pedagógicas como el "Flipped classroom" (o clase invertida), y el "Blended Learning” en un LMS-EVA, que permitirá a los estudiantes de la carrera de Diseño Gráfico, específicamente de la materia de diseño Básico Bidimensional, contar con un entorno sobre el cuál llevar a cabo la práctica pedagógica de sus contenidos de aprendizaje teóricos de manera más adecuada, donde se espera además, como resultado de este proceso de investigación, mejorar significativamente sus relaciones sociales y su rendimiento académico.

La utilización del análisis de redes sociales (ARS), como base metodológica y teórica en el análisis de contextos educativos, se encuentra inicialmente implementado en el contexto ecuatoriano a través de las investigaciones de (Dillon y Espinosa, 2019), en el estudio "Innovación y liderazgo educativo y su influencia en las relaciones sociales y académicas de estudiantes de posgrado"; Dillon, Freire y Espinosa (2019) por medio del estudio: "Interacciones de género en la actividad académica de estudiantes de psicología: revelaciones desde la Teoría de sistemas - RED”; y también, Dillon y Espinosa (2020) a través del estudio: "Relaciones académicas y de género en docentes universitarios y su influencia en la publicación en revistas indexadas de alto impacto". De manera complementaria, se suma este trabajo de investigación, cuyo análisis y reflexiones permitirán a profesionales interesados en la implementación de EVA gamificados en instituciones educativas de cualquier nivel. El contar con marcos teóricos y metodológicos de referencia, ayudará a determinar la influencia de la implementación de estos entornos en los procesos de enseñanza y aprendizaje de sus estudiantes.

Como recomendación, se sugiere considerar la implementación del Entorno Virtual de Aprendizaje "Second Life" como estrategia didáctica gamificada en el proceso de aprendizaje de otras materias, en las que los docentes, por medio del diseño curricular de los componentes de aprendizaje, puedan determinar su adecuada implementación.

También se recomienda considerar la implementación del EVA gamificado denominado "SANSAR", que significa: "Vida cambiante" en lengua Hindi", ya que se constituye como una alternativa mucho más llamativa y actualizada en términos tecnológicos, con una interfaz gráfica y mayor mutabilidad que el entorno virtual "Second Life". A su vez, se debería contemplar la implementación de este tipo de investigaciones en otros contextos y niveles educativos para medir su factibilidad de implementación.

\section{Referencias}

Aguilera, A., Fúquene, C. y Ríos, W. (2014). Aprende jugando: el uso de técnicas de gamificación en entornos de aprendizaje. Revista IM-Pertinente, 2(1), 125-143. https://cutt.ly/IjzgGuw

Albert, R. y Barabási, A. (2002). Statistical mechanics of complex networks. Reviews of modern physics, 74(1), 47-97. https://doi.org/10.1103/RevModPhys. 74.47

Arroyo Vera, Z., Fernández Prieto, S., Barreto Zambrano, L. y Paz Enrique, L. (2018). Entornos virtuales de aprendizaje en comunidades de práctica de docentes universitarios del Ecuador. Revista Ensayos Pedagógicos, 13(2), 185-200. https://doi. org/10.15359/rep.13-2.9 
Cestero, E. y Mateos, A. (2018). Data Science y Redes Complejas: Métodos y Aplicaciones. Editorial Universitaria Ramón Areces.

Delgado, M. y Solano, A. (2009). Estrategias didácticas creativas en entornos virtuales para el aprendizaje. Actualidades Investigativas en Educación, (9)2, 1-21. https://cutt.ly/jjzhRTg

Dillon, F. y Espinosa, D. (2019). Innovación y liderazgo educativo y su influencia en las relaciones sociales y académicas de estudiantes universitarios de posgrado. SATHIRI, 14(2), 138-150. https://doi.org/10.32645/13906925.894

Dillon, F. y Espinosa, D. (2020). Relaciones académicas y de género en docentes universitarios y su influencia en la publicación en revistas indexadas de alto impacto. Ciencia y Educación, 3(3), 7-20. https://doi.org/10.22206/cyed.2019.v3i3. pp7-20

Dillon, F., Freire, I. y Espinosa, D. (2019). Interacciones de género en la actividad académica de estudiantes de psicología: revelaciones desde la Teoría de sistemas - RED. Wimblu, 14(2), 7-25. https://cutt.ly/XjzhBMh

Edel-Navarro, R. (2010). Entornos Virtuales de Aprendizaje. La contribución de "lo virtual" en la educación. Revista Mexicana de Investigación Educativa, 15(44), 7-15. https://cutt.ly/ xjzhzVY

Hernández-Sampieri, R. y Mendoza, C (2018). Metodología de la investigación. Las rutas cuantitativa, cualitativa y mixta. Editorial Mc Graw Hill Education.

Johnson, B. y Onwuegbuzie, A. (2004). Mixed Methods Research: A Research Paradigm Whose Time Has Come [Los métodos de investigación mixtos: un paradigma de investigación cuyo tiempo ha llegado]. Educational Researcher, 33(7), 14-26. https://doi.org/10.3102/0013189X033007014

Martí, P. (2016, marzo 01). El australiano que cambió la forma de aprender en todo el mundo. $\mathrm{La}$ vanguardia. https://url2.cl/zmF7z
Melo-Solarte, D. S. y Díaz, P. A. (2018). El Aprendizaje Afectivo y la Gamificación en Escenarios de Educación Virtual. Información Tecnológica, 29(3), 237-248. https://doi.org/10.4067/ s0718-07642018000300237

Moreno Martínez, N. M., Leiva Olivencia, J. J. y Matas Terrón, A. (2016). Mobile learning, Gamificación y Realidad Aumentada para la enseñanza-aprendizaje de idiomas. IJERI: International Journal of Educational Research and Innovation, (6), 16-34. https://cutt.ly/0jzjnMs

Norman, D. (2001). Elordenador invisible: por qué los buenos productos pueden fracasar, los ordenadores personales son tan complicados y las aplicaciones informáticas son la solución. Paidós Ibérica.

Organización de las Naciones Unidas para la Educación, la Ciencia y la Cultura. (2015). Declaración de Qingdao. Aprovechar las oportunidades digitales. Liderar la transformación de la educación. Estados Unidos.

Padua, J. (2018). Técnicas de Investigación aplicadas a las ciencias sociales. Fondo de Cultura Económica.

Park, J. y Newman, M. (2004). Statistical mechanics of networks. Physical Review, 70(6), 1-15. https:// doi.org/10.1103/PhysRevE.70.066117

Rubido, N., Grebogi, C. y Baptista, M. (2017). Understanding Information Transmission in complex networks, 387(1), 1-5. https://cutt.ly/ OjzjZDp

Saraguro, R., Jara-Roa, D. I., Agila-Palacios, M. y Sarango-Lapo, P. (2015). Uso de técnicas de gamificación en el Diseño Tecnopedagógico de un MOOC. Universidad Técnica Particular.

The University of British Columbia. (1995, 04 10). Murray Golberg develops WebCT. Computer Science - People and Milestones, pág. 1.

Torres, A. y Romero, L. (2018). Gamificación en Iberoamérica. Experiencias desde la Comunicación y la Educación. Editorial Abya. https://cutt. ly/Kjzj3xM 
UNESCO (2008). Estándares de competencia en TIC para docentes. UNESCO.

Velázquez, A. y Aguilar, N. (2005). Manual introductorio al análisis de redes sociales. Revista Redes, 29(1), 16-43. https://cutt.ly/PjzktaZ

Villarreal, M. (2018). El diseño de entornos digitales de aprendizaje. (Documento inédito). https:// cutt.ly/tjzky6j
Villavicencio, E. (2016, abril 06). Mundos virtuales: Second Life como aporte a la educación. Grupo Avatar. https://cutt.ly/Ujzkh2E

Watts, D. (2006). A seis grados de separación. Paidós Ibérica, S. A. 